ARTICLE

Received 2 Mar 2015 | Accepted 10 Oct 2015 | Published 16 Nov $2015 \quad$ DOl: 10.1038/ncomms9879 OPEN

\title{
Spindle assembly checkpoint inactivation fails to suppress neuroblast tumour formation in aurA mutant Drosophila
}

Renaud Caous ${ }^{1}$, Aude Pascall, Pierre Romé ${ }^{1, \dagger}$, Laurent Richard-Parpaillon ${ }^{1}$, Roger Karess ${ }^{2}$ \& Régis Giet ${ }^{1}$

Tissue homeostasis requires accurate control of cell proliferation, differentiation and chromosome segregation. Drosophila sas-4 and aurA mutants present brain tumours with extra neuroblasts (NBs), defective mitotic spindle assembly and delayed mitosis due to activation of the spindle assembly checkpoint (SAC). Here we inactivate the SAC in aurA and sas- 4 mutants to determine whether the generation of aneuploidy compromises NB proliferation. Inactivation of the SAC in the sas- 4 mutant impairs NB proliferation and disrupts euploidy. By contrast, disrupting the SAC in the aurA mutant does not prevent NB amplification, tumour formation or chromosome segregation. The monitoring of Mad2 and cyclin B dynamics in live aurA NBs reveals that SAC satisfaction is not coupled to cyclin B degradation. Thus, the NBs of aurA mutants present delayed mitosis, with accurate chromosome segregation occurring in a SAC-independent manner. We report here the existence of an Aurora A-dependent mechanism promoting efficient, timed cyclin B degradation.

\footnotetext{
${ }^{1}$ Institut de Génétique et Développement de Rennes-Université de Rennes I-CNRS- UMR 6290, 2 avenue du Pr Léon Bernard, 35043 Rennes, France. 2 Institut Jacques Monod-Université Paris Diderot-Paris 7, 15 rue Hélène Brion, 75205 Paris, France. †Present address: The Wellcome Trust Centre for Cell Biology, School of Biological Sciences, University of Edinburgh, Michael Swann Building, Max Born Crescent, Edinburgh EH9 3BF, UK. Correspondence and requests for materials should be addressed to R.G. (email: regis.giet@univ-rennes1.fr).
} 
T he mitotic spindle is a microtubule-based structure involved in the segregation of the two sets of duplicated chromosomes in the two daughter cells. In polarized fly neural stem cells (neuroblasts (NBs)), it is also involved in the differential segregation of cell fate determinants anchored to the apical and basal cell cortex. In this type of cell division, the two daughter cells have different fates: one retains NB identity and continues to proliferate, whereas the other differentiates. Defects in spindle orientation lead to the abnormal delivery of cell fate determinants. Consequently, both daughter cells may become NBs, leading to tumour formation ${ }^{1-3}$. This is the case for sas-4 mutant flies, which have no centrosomes and display severe spindle orientation defects ${ }^{4,5}$. Spindle orientation and assembly are therefore tightly linked in the maintenance of tissue homeostasis and euploidy in the fly central nervous system (CNS). An understanding of the mechanisms involved in the defective asymmetric division of NBs is therefore relevant to cancer stem cell research ${ }^{1}$.

Aurora A kinase has been implicated in the formation of human cancers and is considered to be an oncogene $e^{6}$. Paradoxically, Drosophila Aurora A can also act as a tumour suppressor during the asymmetric division of $\mathrm{NBs}^{7-9}$. Mutations of Drosophila aurA result in the production of abnormal mitotic spindles with defective astral microtubule attachments to the apical cortex, leading to spindle misorientation, abnormal daughter cell fate acquisition and expansion of the NB population ${ }^{7-11}$. The transplantation of mutant aurA brains into the abdomens of host flies leads to tumour formation ${ }^{5}$. The spindle defects of aurA mutant NBs also lead to delays in mitotic progression. Wild-type NBs exit mitosis $\sim 6-7 \mathrm{~min}$ after nuclear envelope breakdown (NEBD), whereas aurA hypomorphic mutant neuroblasts do not exit mitosis until $\sim 20 \mathrm{~min}$ after NEBD. This delay is presumably mediated by prolonged spindle assembly checkpoint (SAC) activation in response to incorrectly attached kinetochores ${ }^{4,7,12}$

In human cancer cells, weakening of the SAC compromises chromosome segregation and leads to massive aneuploidy and cell death ${ }^{13}$. We show here that the absence of the SAC in sas-4 mutants strongly impairs chromosome segregation, euploidy and the ability of neural tissues to proliferate and to induce tumours. However, chromosome segregation and cell ploidy are unaffected by the absence of the SAC in aurA mutants, and the mutant tissue can still induce tumours following their injection into host flies. Our results suggest that impaired cyclin B degradation compensates for the defect in chromosome segregation in aurA neural tissues, in the absence of the SAC.

\section{Results}

SAC inactivation does not impair aurA NB amplification. We investigated the effects on fly viability and brain development of an absence of the SAC in aurA ${ }^{8839}$ null flies (hereafter referred as aurA). Mad2 is a key component of the SAC apparatus and appears to have no other role in Drosophila ${ }^{14}$

We therefore created various double-mutant lines for aurA $A^{8839}$ and $m a d 2$, and checked the double mutation by confirming that the Mad2 protein was absent and that there was a higher mobility form of the truncated AurA $\mathrm{K}^{\mathrm{K} 77 / \text { stop }}$ mutant protein (Fig. 2c, second panel from the top). We confirmed that the null $\operatorname{mad} 2^{P}$ mutant (hereafter referred to as mad2) was viable ${ }^{4,14}$. In mad2 larval brains, the number of NBs ( \pm s.d.) $(73.2 \pm 9.0 \mathrm{NBs}$ per lobe, $n=22)$ was similar to that in the wild type $(75.4 \pm 10.5$ NBs per lobe, $n=14$ ) (Fig. 1a left panels and b).

Surprisingly, the aurA mad2 double mutant displayed marked brain overgrowth, similar to that observed for aurA mutants (Supplementary Fig. 5a). Consistent with the overgrowth phenotype, the larval brains of both aurA and aurA mad2 individuals had more NBs per lobe than the WT (Fig. 1a right panels and c). We labelled larval brain tissues of single- and double-mutant brains with H2A-green fluorescent protein (GFP), dissected them and injected them into host flies to assess their ability to induce tumour formation ${ }^{15}$. Tumour formation rates were similar for aurA mad2 double-mutant brains ( 80\%, 45/56 injected flies, Fig. 1e) and for aurA single-mutant brains ( $82 \%$, 41/50). By contrast, neither WT nor mad2 mutant brains triggered tumour formation, consistent with published findings ${ }^{5}$. We confirmed these results with another aurA allelic combination (aurA ${ }^{8839} /$ aurA $^{17961}$ ), and with a bubR1 allele causing a defective SAC (hereafter named bubR1-KEN (ref. 16)). Like aurA mad2 brain tissues, bubR1-KEN aurA ${ }^{8839}$ / aurA ${ }^{17961}$ brains were bigger than WT, contained large numbers of NBs and had high mitotic indices (Supplementary Fig. 1).

We investigated whether the ability of aurA neural tissues to develop tumours even in the absence of a SAC was a general feature of mutants generating supernumerary NBs, by studying the consequences of inactivating the SAC in sas- 4 mutants. The spindles of sas-4 mutants lack centrioles and astral microtubules, and they display alignment defects similar to those seen in aurA mutants $^{4,10,17}$ (Fig. 1d). In sas-4 NBs, spindles assemble from microtubules that have randomly nucleated around chromatin, and the duration of mitosis is prolonged by $30-40 \%$, presumably due to the action of the SAC ${ }^{4}$. Thus, while sas- 4 mutants display very low levels of aneuploidy, their spindle orientation is defective and their brains can generate tumours on transplantation ${ }^{5}$.

We confirmed that the sas- $4^{s 2214}$ mutant (hereafter referred to as sas-4) was viable ${ }^{4,14}$. The brains of sas-4 mutants contained about $70 \%$ more NBs than WT brains $(126.2 \pm 16.2$ per lobe, $n=18, P=7.9 \times 10^{-8}$, Wilcoxon test), consistent with their ability to develop into tumours when injected into host flies ${ }^{5}$ (Fig. 1f). However, double-mutant sas-4 mad2 individuals had much smaller brains, with far fewer NBs $(42.0 \pm 14.5$ NBs per lobe, $n=14$; Fig. 1a,b), and their brain tissues did not form tumours after injection into host flies (Fig. 1f). In addition, dividing NBs were only very rarely observed in the double mutants, and almost all these cells had abnormal spindle structures (Fig. 1d). Similarly, a bubR1-KEN sas-4 double mutant was found to have impaired tissue growth and smaller than normal numbers of NBs (Supplementary Fig. 2a,b).

High mitotic indices and normal ploidy in aurA mad2 brains. Inactivation of the SAC in sas-4 mutants severely impaired brain development and triggered spindle assembly failure, whereas such inactivation had little effect in aurA mutants. The growth defects in the sas-4 mad2 mutant were associated with the presence of numerous aneuploid $(42.6 \%)$ and polyploid (35.5\%) cells. By contrast, the rates of aneuploidy and polyploidy were only slightly increased by SAC ablation in aurA mutants (Supplementary Fig. 3). These opposite results for sas-4 and aurA mutant NBs suggest that a specific mechanism operates in aurA mutant NBs (but not in sas-4 mutant NBs), allowing correct chromosome segregation to occur even if the SAC is not functional.

The larval brains of aurA mutant individuals have a high mitotic index $(6.7 \% \text {, versus about } 1 \% \text { in the WT })^{11}$ (Fig. 2a). This high mitotic index is presumably due to the intervention of the SAC, because aurA mutant spindle assembly is defective. Surprisingly, however, aurA mad2 double mutants had a mitotic index of $5.8 \pm 2.8 \%$, very similar to that of aurA brains (Fig. 2a). As described previously, aurA mitotic NBs divide symmetrically to generate smaller $\mathrm{NBs}^{7}$. In addition, the mitotic cells of both aurA and aurA mad2 individuals presented highly 

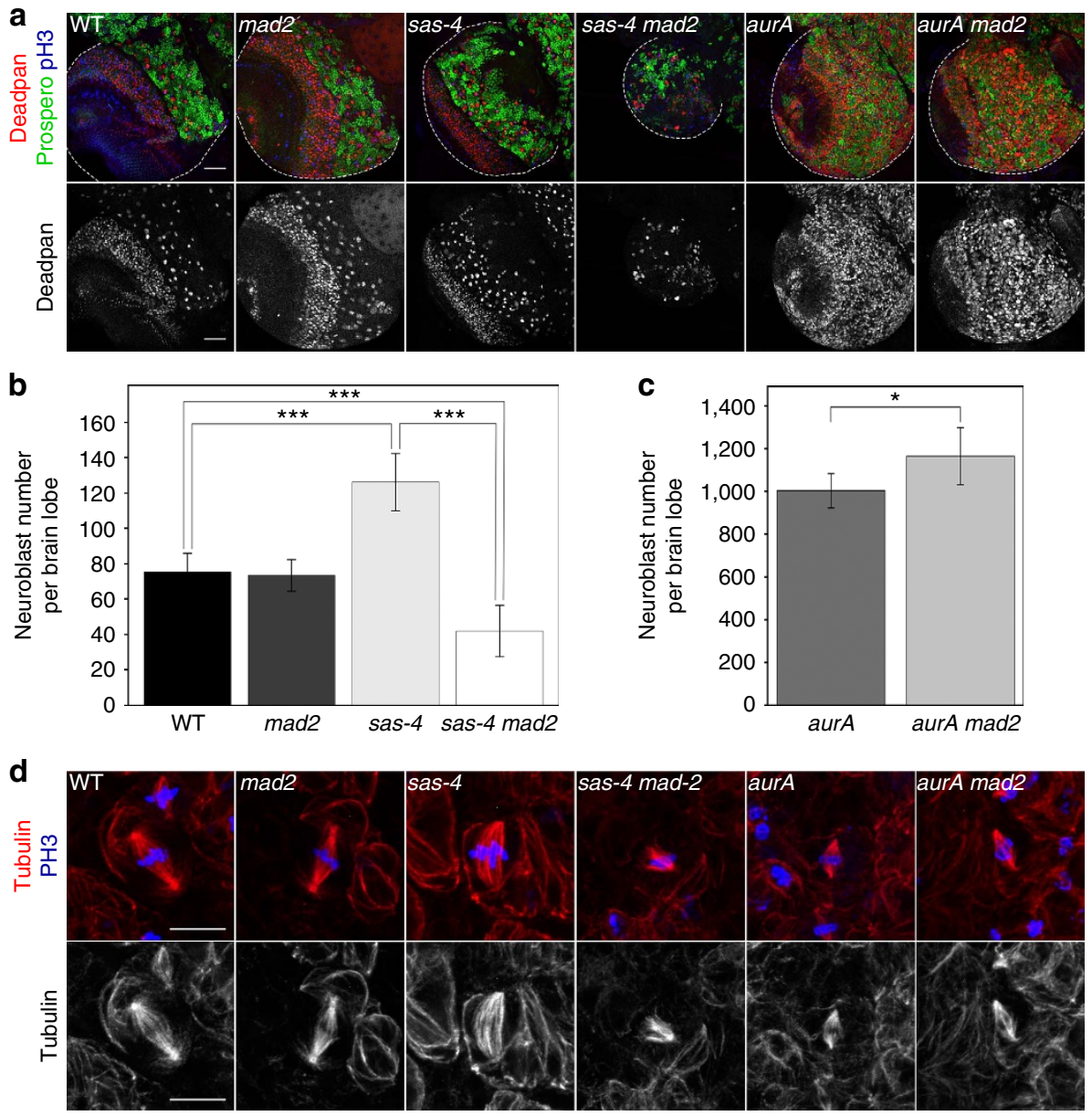

$\mathbf{e}$
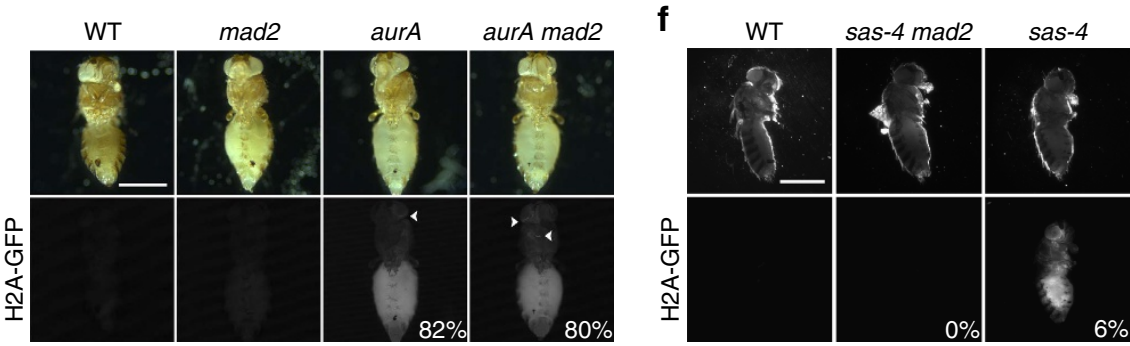

Figure 1 | SAC deletion prevents the amplification of sas-4 mutant NBs but not of aurA mutant NBs. (a) Examination of WT, sas-4, aurA, mad2, sas-4 mad2 and aurA mad2 mutant brain development. WT and mad2 mutant brains grew normally, whereas sas-4 and aurA mutant brains contained large numbers of NBs. SAC deletion impaired brain development in combination with sas-4, but not with aurA. The pan-NBs were counted by staining of the central brain region with an anti-deadpan antibody (red and lower monochrome panels). Staining for the neuronal marker prospero is shown in green. Staining for phosphorylated histone $\mathrm{H3}$ (Ser10) is shown in blue. Scale bar, $50 \mu \mathrm{m}$. (b) Quantification of pan-NBs ( \pm s.d.) in WT, sas-4, mad2 and sas-4 mad2 brain lobes. WT and mad2 brain lobes had $75.4 \pm 10.5$ NBs per lobe $(n=14)$ and $73.2 \pm 9.0$ NBs per lobe $(n=22)$, respectively. sas-4 lobes contained a larger number of NBs per lobe $(126.2 \pm 16.2 \mathrm{NBs} ; n=18)$. The disruption of Mad2 in a sas-4 background decreased the growth rate and number of NBs $(42.0 \pm 14.5, n=14) .{ }^{\star \star \star} P<10^{-10}$ (Wilcoxon test). (c) Quantification of pan-NBs ( \pm s.d.) in aurA and aurA mad2 brain lobes. The number of NBs in aurA brain lobes (1003.6 $\pm 79.7, n=12$ ) was high, even in the absence of Mad2 (1164.2 $\pm 134.3, n=11) .{ }^{\star} P<3 \times 10^{-3}$ (Wilcoxon test). (d) SAC deletion compromises spindle morphology in sas -4 but not aurA mutant NBs. Metaphase NBs from the indicated genotypes were stained for phosphorylated histone H3 (Ser10) (blue), tubulin (red and lower panels in monochrome). Many spindles in sas-4 brains were bipolar, but sas-4 mad2 mutant spindles were abnormal in shape. The aurA and aurA mad 2 mutants had short bipolar spindles. Scale bar, $10 \mu \mathrm{m}$. (e) The ability of aurA mutant brains to develop tumours is not compromised by SAC deletion. WT, aurA, mad2 and aurA mad2 brains were labelled with H2A-GFP and transplanted into host flies to assess their tumorigenic potential ${ }^{15}$. The transplantation of aurA and aurA mad2 brain tissues induced tumour formation in $82 \%(41 / 50)$ and $80 \%(45 / 56)$ of cases. (f) The transplantation of H2A-GFP-labelled sas-4 brain tissues led to tumour formation $(5.9 \%, 6 / 102)$ whereas the transplantation of sas-4 mad2 brain tissues did not $(0 \%, 0 / 112)$. Scale bar, $0.5 \mathrm{~mm}$.

condensed chromosomes with preserved centromeric cohesion (Fig. 2b), a feature characteristic of a prolonged $M$ phase ${ }^{18}$. Cyclin A protein levels were normal (Fig. 2c, right) and this protein was degraded normally, during prometaphase, in all genotypes examined (Fig. 2d). However, phosphorylated histone (phospho-histone) H3 (Ser10) and cyclin B protein levels were higher in aurA mad2 and aurA brain extracts than in WT or mad2 extracts (Fig. 2c, left). Analyses of fixed specimens from 
a

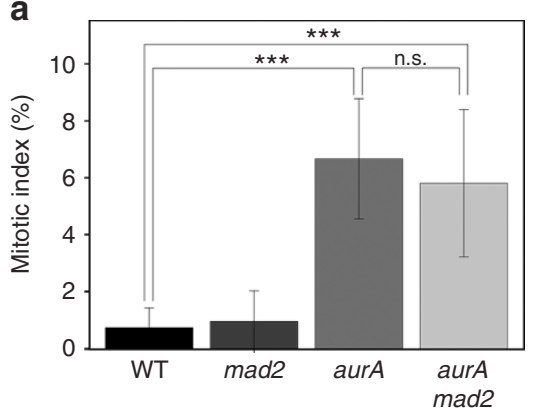

b

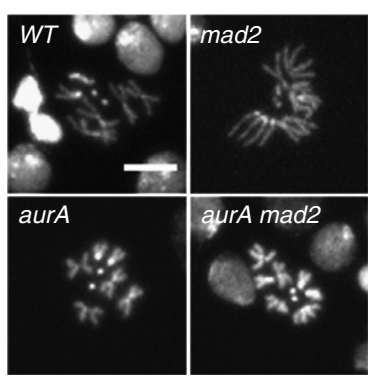

C
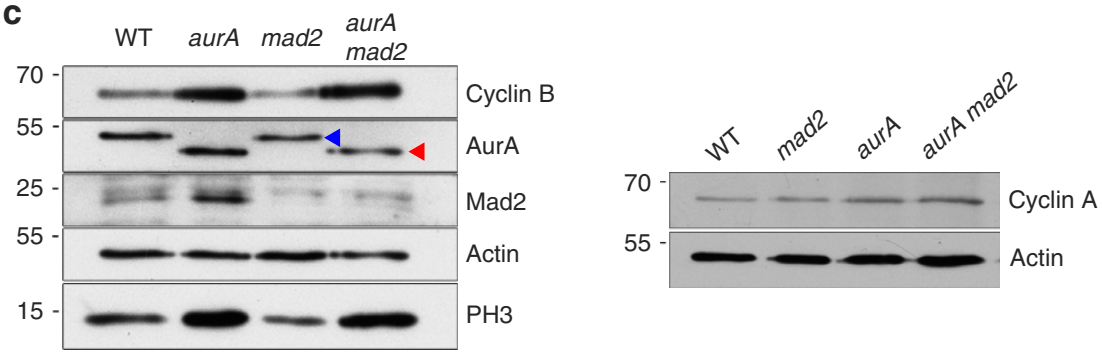

d
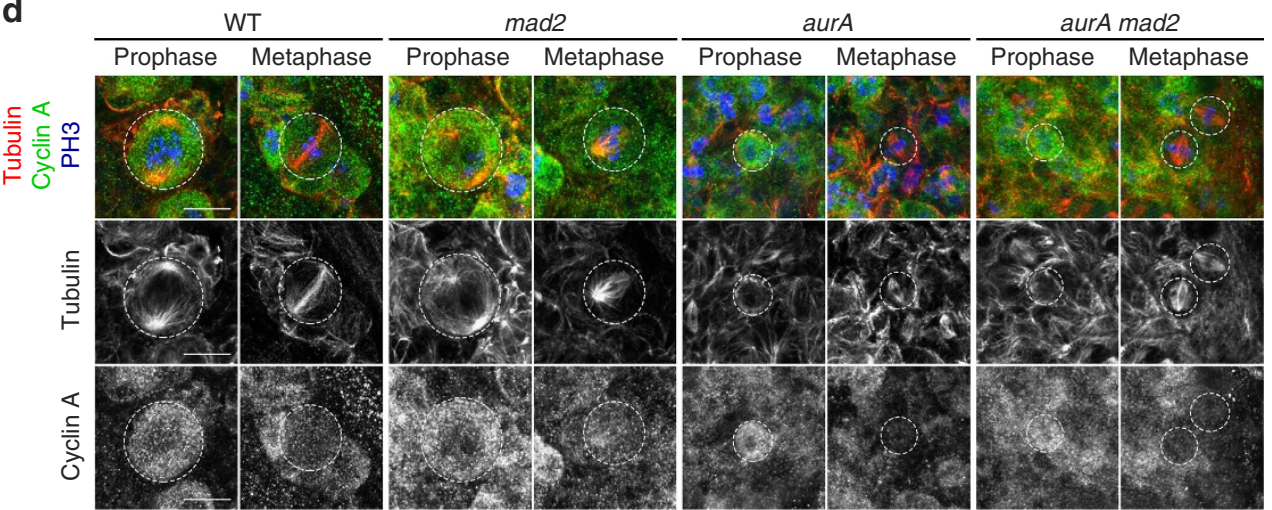

e
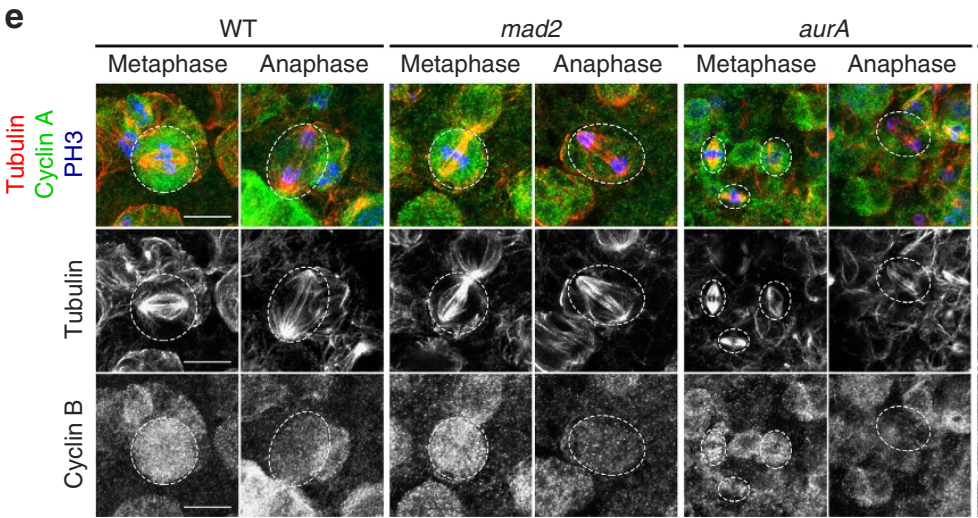

aurA mad2

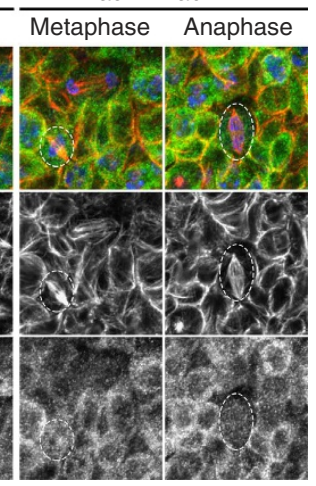

Figure 2 | Mitotic delay in aurA mutants is SAC independent. (a) Analysis of mitotic indices ( \pm s.d.) in WT, mad2, aurA and aurA mad2 neural tissues. WT: $0.73 \pm 0.69 \%(n=11,332$, three brains); $\operatorname{mad2}: 0.96 \pm 1.07 \%(n=9,507$, three brains); aurA: $6.7 \pm 2.1 \%$ ( $n=11,334$, three brains); and aurA mad2: $5.8 \pm 2.8 \%$ ( $n=8,648$, three brains). ${ }^{\star \star \star} P<10^{-10}$ (Wilcoxon test). (b) Chromosome morphology in WT, mad2, aurA and aurA mad2 neural tissues. Note that in both aurA (bottom left) and aurA mad2 (bottom right) mutants, the mitotic cells have highly condensed chromosomes, indicative of a delay in mitosis, unlike WT and mad2 mutant brain cells (top). Scale bar, $10 \mu \mathrm{m}$. (c) Western blots showing the levels of cyclin B, AurA, Mad2, phospho-histone H3 (Ser10) (left) and cyclin A (right). Actin was used as a loading control. Cyclin B and phospho-histone H3 (Ser10) are high in aurA and aurA mad2 neural tissue extracts, whereas cyclin A protein levels are not. The truncated AurA $\mathrm{K}^{\mathrm{K} 77 / \mathrm{STOP}}$ corresponding to the aurA $\mathrm{A}^{8839}$ mutation is detected as a higher mobility protein in the corresponding aurA and aurA mad2 brain extracts (red triangle), whereas the endogenous Aurora $\mathrm{A}$ is indicated by the blue triangle. (d) Cyclin A degradation occurs during prometaphase in WT, mad2, aurA and aurA mad2 mitotic cells. WT and mutant brains of the indicated genotypes were fixed and stained for cyclin A (green and lower monochrome panels) during G2 and prometaphase. Microtubules are labelled in red (and on the middle monochrome panels) and phospho-histone $\mathrm{H} 3$ (Ser10) is displayed in blue. (e) Cyclin B degradation occurs at the metaphase to anaphase transition in WT, mad2, aurA and aurA mad2 mutant brains. Brains were fixed and stained for cyclin B (green and lower monochrome panels), tubulin (red and middle monochrome panels) and phospho-histone H3 (Ser10). Scale bar, $10 \mu \mathrm{m}$. 
aurA and aurA mad2 mutants confirmed the high frequency of mitotic cells labelled for phospho-histone H3 (Ser10) and cyclin B. However, cyclin B was ultimately degraded in all anaphase cells examined, for both genotypes ( $n>20$; Fig. 2e). Finally, we observed no lagging chromosomes during anaphase and telophase in fixed preparations of tissues from aurA and aurA mad2 mutants $(n>40)$. These findings suggest that mitotic exit and cyclin $\mathrm{B}$ degradation were delayed in a SAC-independent manner in aurA mad2 mutant NBs, and that this delay was sufficient to allow correct attachment of the chromosomes to the spindle before the onset of anaphase.

A delay between SAC satisfaction and anaphase in aurA NBs. We characterized this unexpected phenotype further by assessing SAC activation by following GFP-Mad2 recruitment and the timing of cyclin B degradation in live aurA mutant NBs. The mitotic delay was very long $(\sim 60 \mathrm{~min})$ for the $a u r A^{8839}$ allele. We therefore also used a combination of less severe alleles (aurA $A^{14641}$, aur ${ }^{17961}$ ) (ref. 7), making it possible to minimize the bleaching of red fluorescent protein (RFP)-Histone H2A and GFP-tagged proteins during the time course of the experiments (Fig. 3a,b). In WT NBs, GFP-Mad2 labelled the nuclear envelope and nucleoplasm before mitosis, and weak fluorescence was observed at the kinetochores during prometaphase (Fig. 3d and Supplementary Movie 1), consistent with previous findings ${ }^{19,20}$. In all WT cells examined, this weak GFP-Mad2 signal at the kinetochores completely disappeared $1.76 \pm 0.85 \mathrm{~min} \quad(n=55)$ before anaphase onset (Fig. 3c). In aurA ${ }^{14641} /$ aurA ${ }^{17961}$ mutant NBs, GFP-Mad2 strongly labelled the kinetochores, consistent with defective kinetochore-microtubule attachment and strong SAC activation (Fig. 3a,b,e and Supplementary Movie 2). This strong GFP-Mad2 kinetochore signal decreased slowly in intensity as the chromosomes congressed to the metaphase plate, indicating a delay in the establishment of correct kinetochore-microtubule attachments (Fig. 3e, top and Supplementary Movie 2). However, the time lag between the disappearance of GFP-Mad2 from the kinetophores and the onset of anaphase was significantly longer $(5.75 \pm 1.88 \mathrm{~min}, n=22)$ in all dividing aurA ${ }^{14641} /$ aur $A^{17961}$ cells than in WT cells (Fig. 3c).

Cyclin B degradation is impaired in aurA mutant NBs. Anaphase onset is directly connected to anaphase promoting complex/cyclosome (APC/C) activation and the degradation of mitotic targets, including cyclin $\mathrm{B}$ and securin, therefore we monitored cyclin B-GFP degradation in aurA and aurA mad2 mutants. Consistent with previous findings, cyclin B-GFP accumulated at the centrosome and in the nucleus of WT NBs before entry into mitosis. Following NEBD, cyclin B-GFP was visible throughout the cell, but the fluorescence was particularly intense in the kinetochore region and mitotic spindle ${ }^{19,21}$ (Fig. 4a and Supplementary Movie 3). The cyclin B-GFP signal began to disappear abruptly, shortly after the last chromosome reached the metaphase plate, and half the cyclin B-GFP present was degraded $4.75 \pm 1.04 \mathrm{~min}(n=18)$ after metaphase plate formation (Fig. 4e). The cells of the aurA ${ }^{14641} /$ aurA ${ }^{17961}$ mutant took $11.3 \pm 3.5 \mathrm{~min}(n=23)$ to degrade half their cyclin B (Fig. $4 \mathrm{~b}, \mathrm{e}$ and Supplementary Movie 4), whereas aurA null mitotic cells took $46.2 \pm 12.6 \mathrm{~min}(n=12$; Fig. 4 d,e and Supplementary Movie $6)$. The introduction of the mad2 mutation in the aurA null mutant shortened the time required for half the cyclin $B$ to be degraded to $23.2 \pm 8.9 \mathrm{~min}(n=17)$ but did not restore the WT timing of mitosis (Fig. 4c,e and Supplementary Movie 5). Consistent with observations in fixed anaphase specimens, live imaging of aurA and aurA mad2 mutant cells showed no lagging/ delayed chromatids during anaphase that might have resulted from incorrect attachment ${ }^{9,22}$. This suggests that the machinery responsible for correcting inappropriate attachments between kinetochores and spindle microtubules is fully functional. As cells with Aurora A defects display delayed mitosis, we investigated whether AurA overexpression could accelerate mitosis, as in some cancer cell lines ${ }^{23}$. However, we found that mitotic timing was normal under normal conditions in NBs overexpressing Aurora A kinase, with no acceleration (Supplementary Fig. 4). Furthermore, these NBs remained arrested in $M$ phase in the presence of microtubule depolymerizing drugs (unpublished observations).

aurA mutation restores the euploidy of sas-4 mad2 brains. Our results suggest that aurA mutant NBs display defective cyclin B degradation, resulting in a SAC-independent delay of mitosis. If this is indeed the case, then introducing the aurA mutation in a sas- 4 mad 2 background should (to some extent) restore mitotic timing and euploidy (by lengthening the $M$ phase and correcting the defective kinetochore/microtubule attachments). Consistent with this hypothesis, we found that the mitotic index increased from $0.6 \%$ in the sas $-4 \mathrm{mad} 2$ mutant to $4.7 \%$ in the aurA sas- 4 mad2 triple mutant (Supplementary Fig. 5d). In addition, only $21.9 \%$ of the cells had the correct number of chromosomes in sas-4 mad2 double mutants, whereas most of the mitotic aurA sas-4 mad2 cells (63.9\%) displayed normal ploidy (Supplementary Fig. 3c). Furthermore, brain overgrowth and NB amplification were also detected in this triple-mutant background (Supplementary Fig. 5a-c).

\section{Discussion}

This report suggests that Aurora A may be required for the correct APC/C-dependent degradation of cyclin B. The SAC is transiently activated in aurA mutants (as shown by the strong recruitment of GFP-Mad2 to kinetochores), but this is not

Figure 3 | Metaphase to anaphase transition is delayed in aurA hypomorphic mutant NBs. (a) Mitotic timing and spindle assembly checkpoint analysis in 26 WT (left) and 22 aurA ${ }^{14641} /$ aurA ${ }^{17961}$ NBs (right). The grey and white bars indicate the time spent by each cell in prometaphase and metaphase, respectively. The green bar indicates the time for which GFP-Mad2 was present at the kinetochores. (b) Box plots showing prometaphase duration (left, WT: $3.27 \pm 0.93$ min; aurA ${ }^{14641} /$ aurA ${ }^{17961}: 8.81 \pm 3.42 \mathrm{~min} ; P=1.2 \times 10^{-11}$, Wilcoxon test), metaphase duration (middle, WT: $3.7 \pm 1.3$ min; aurA ${ }^{14641} /$ aurA ${ }^{17961}: 11.6 \pm 3.8 \mathrm{~min} ; P=1.0 \times 10^{-11}$, Wilcoxon test) and Mad2 occupancy time at the kinetochore (right, WT: $5.2 \pm 1.1 \mathrm{~min}$; aurA ${ }^{14641} /$ aurA ${ }^{17961}$ : $14.6 \pm 5.4$ min; $P=3.0 \times 10^{-13}$, Wilcoxon test) in WT $(n=54)$ and aurA $A^{14641} /$ aur $A^{17961}(n=22)$ NBs. The above values are the means \pm s.d. Box plot: boxes show the upper and lower quartile. $P$ values are determined with a Wilcoxon test. (c) Box plots showing the time interval between $S A C$ satisfaction and mitotic entry. In WT cells, the time between SAC satisfaction and anaphase onset was $1.76 \pm 0.94$ min $(n=54)$. By contrast, this interval was much longer, and significantly so, in the aurA mutant, at $5.75 \pm 1.88 \mathrm{~min}(n=22)$. ${ }^{\star \star \star} P=1.9 \times 10^{-10}$, Wilcoxon test. Box plot: boxes show the upper and lower quartile. $P$ values are determined with a Wilcoxon test. (d,e) RFP-histone H2A was used to monitor chromosome dynamics (red in the top panels and in the monochrome middle panels), and GFP-Mad2 (green and bottom lower monochrome panels) was used to monitor chromosome attachment and SAC satisfaction. Scale bars, $10 \mu \mathrm{m}$. Time (min:s) is indicated at the top left of each image. NEBD began at 00:00. (d) Analysis of chromosome and GFP-Mad2 dynamics in WT NBs. (e) Analysis of chromosome and GFP-Mad2 dynamics in aurA $A^{14641} /$ aurA ${ }^{17961}$ NBs. 
sufficient to account for the observed delay in mitosis. Our genetic and live imaging studies indicate that the delay in aurA mutant cells has two components, one dependent on the SAC and the other independent of the SAC. In Drosophila, mutations affecting APC/C subunits lead to poor CNS development and an accumulation of the mitotic cyclins $A$ and $B^{24,25}$. Cyclin A levels are normal and this protein is degraded normally in aurA mutant tissues. It is therefore unlikely that overall APC/C activity is affected. Instead, we favour the hypothesis that some, but not all, APC/C substrates (the most important of which being cyclin B) are more stable in aurA and are not efficiently ubiquitylated. The underlying mechanism remains unclear, but one recent report a

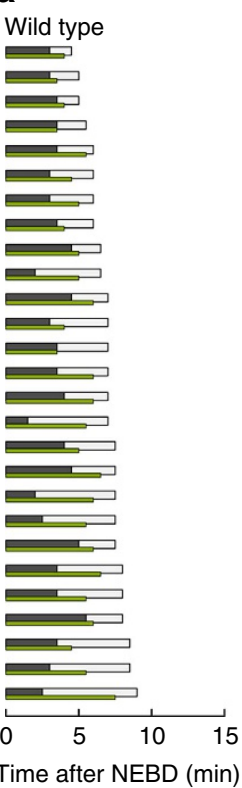

$\operatorname{aurA}^{14641} /$ aurA $A^{17961}$
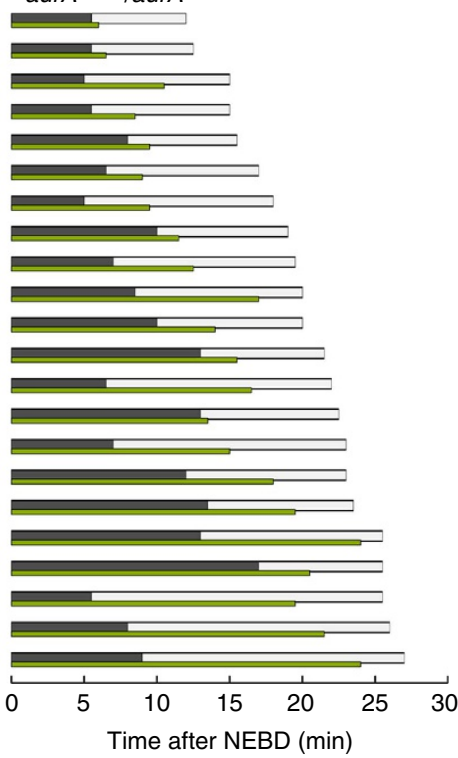

Prometaphase $\square$ Metaphase

$\square$ GFP-Mad2 residency time b

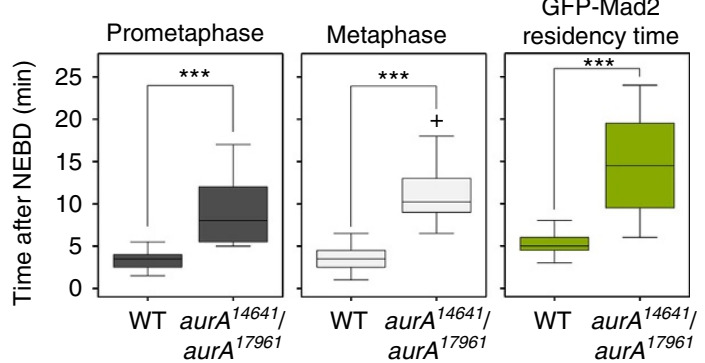

C

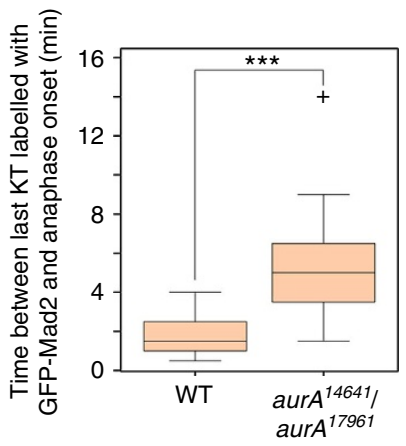

d

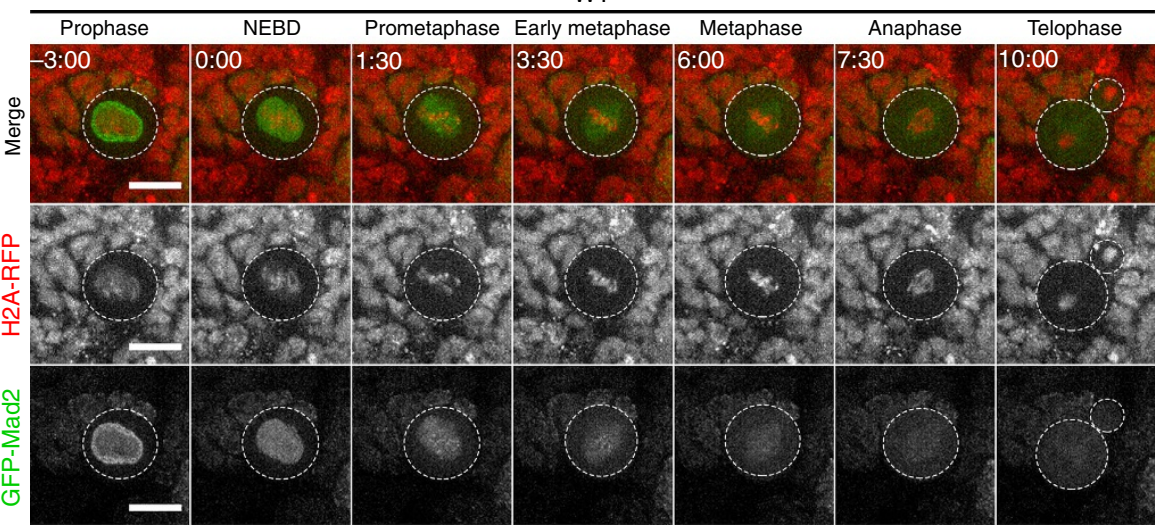

e $\operatorname{aur}^{14641} / \operatorname{aur}^{17961}$

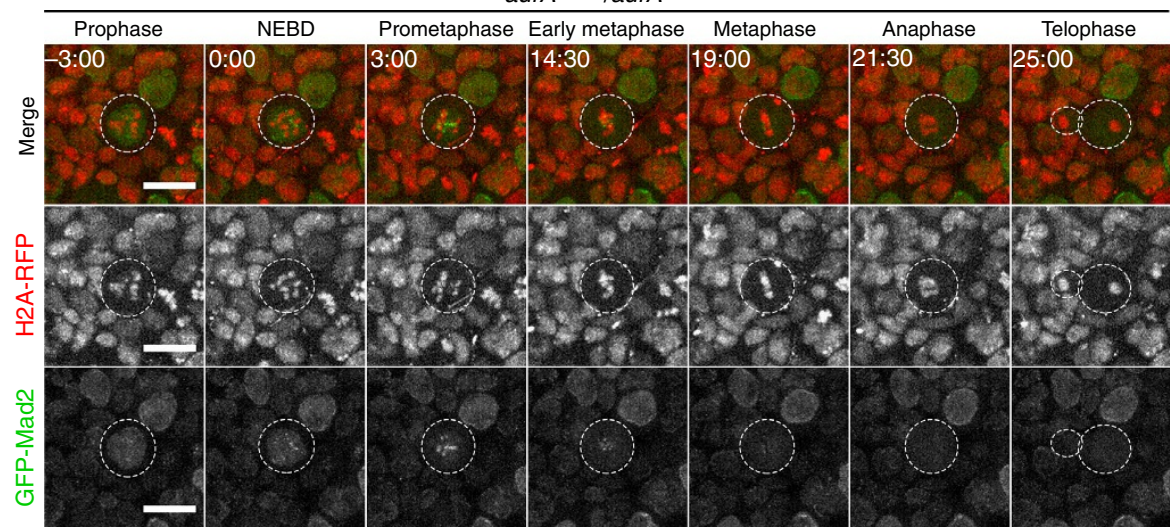


a

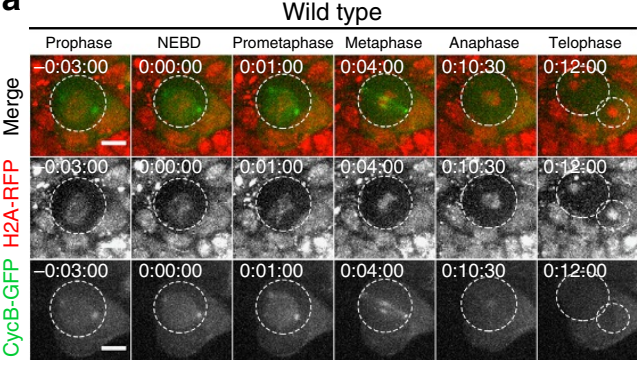

b

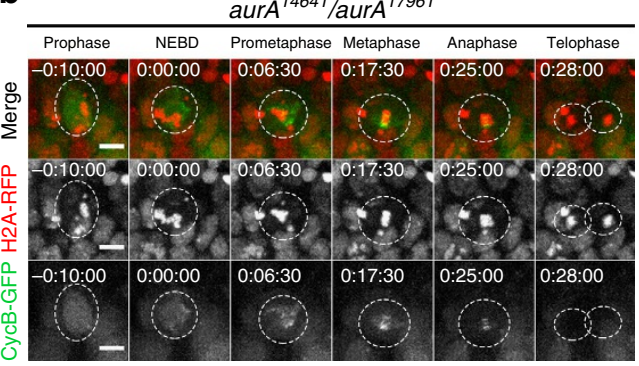

C

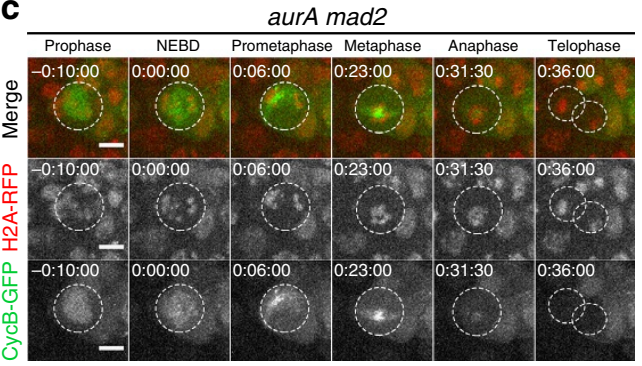

d

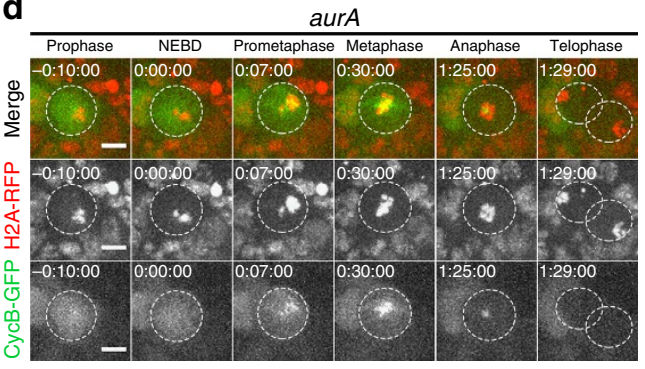

e

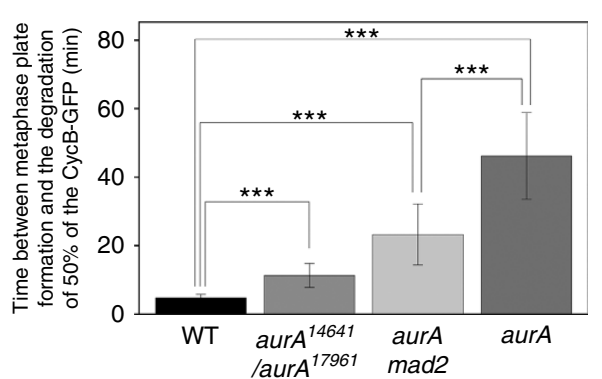

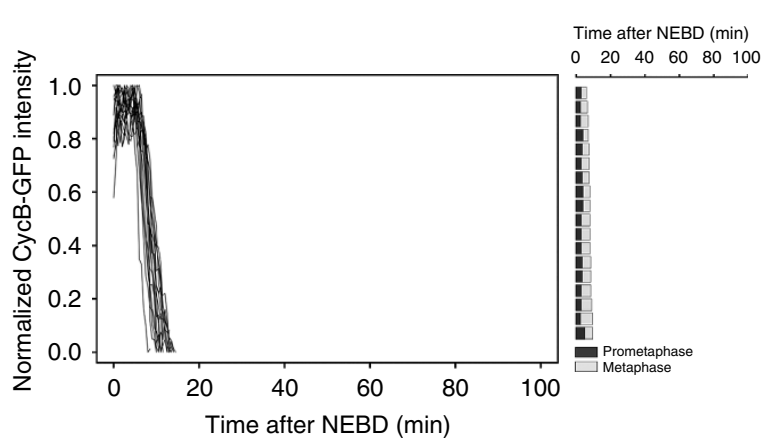

Time after NEBD ( $\mathrm{min})$
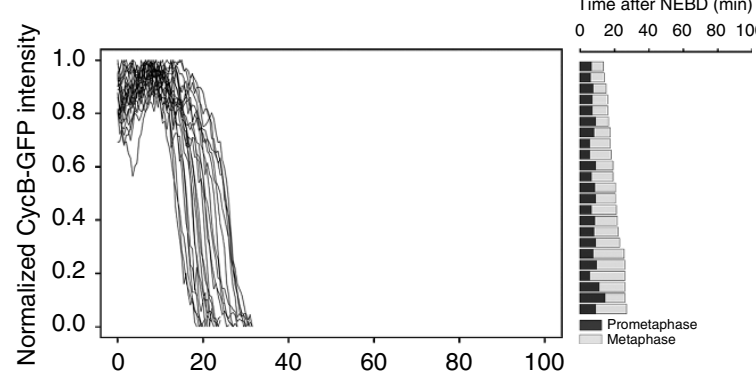

$\begin{array}{llllll}0 & 20 & 40 & 60 & 80 & 100\end{array}$

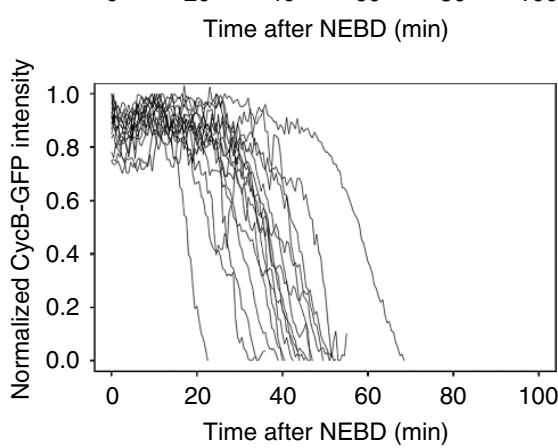

Time after NEBD $(\mathrm{min})$ $\begin{array}{llllll}20 & 40 & 60 & 80 & 100\end{array}$

政

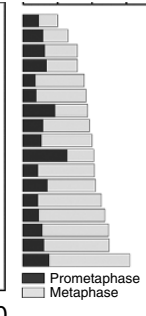

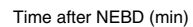
$0 \quad 20 \quad 40 \quad 60 \quad 80 \quad 100$
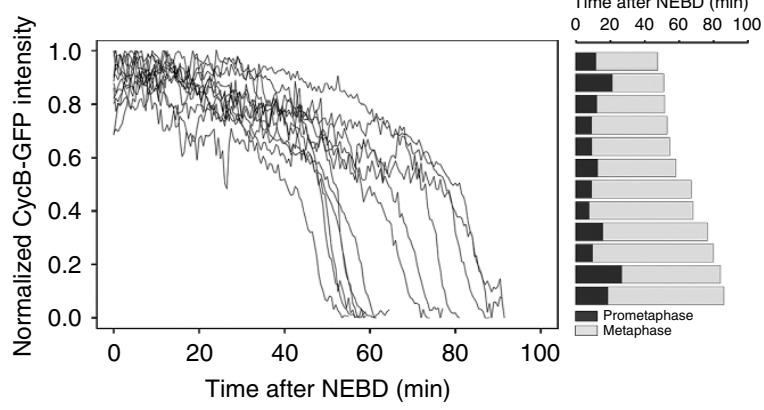

Figure 4 | Cyclin B degradation is delayed in a SAC-independent manner in aurA NBs. (a-d) (Far left column panels) RFP-histone H2A was used to monitor chromosome dynamics (red in the top panels and in the middle monochrome panels), and cyclin B-GFP was used to determine the amounts of cyclin B protein in individual cells (green and lower monochrome panels). The panels of the middle column show the cyclin B-GFP degradation profiles of the corresponding genotypes. The timing of mitosis (far right column panels) is indicated for each individual NB. The black and grey bars indicate the durations of prometaphase and metaphase, respectively. Time (h:min:s) is indicated at the top left of each image. NEBD began at 00:00:00. Scale bars, $5 \mu \mathrm{m}$. (a) Timing of mitosis and cyclin B-GFP degradation in WT NBs $(n=18)$. (b) Timing of mitosis and cyclin B-GFP degradation in aurA $A^{14641} /$ aurA ${ }^{17961} \mathrm{NBs}(n=23)$. (c) Timing of mitosis and cyclin B-GFP degradation in aurA mad2 double-mutant NBs $(n=17)$. (d) Timing of mitosis and cyclin B-GFP degradation in aurA null mutant NBs $(n=12)$. (e) Graph showing the mean time ( \pm s.d.) to $50 \%$ cyclin B-GFP degradation for WT, mad2, aurA mad2 and aurA NBs. WT: $4.8 \pm 1.1 \mathrm{~min}(n=18)$; AurA ${ }^{14641} /$ aurA ${ }^{17961}: 10.3 \pm 3.4 \min (n=23)$; aurA: $46.2 \pm 12.6(n=12)$; and aurA mad2: $23.3 \pm 8.9 \min (n=17)$. ${ }^{\star \star \star} P<7 \times 10^{-5}$ (Wilcoxon test). 
revealed that Drosophila cyclin B is itself a substrate of Aurora B kinase during abscission, raising the possibility that cyclin B could be a target of Aurora A kinase. However, it seems unlikely that such phosphorylation events could control the correct timing of mitosis, as mitotic timing was found to be normal in dividing germline cells expressing non-phosphorylatable version of cyclin $\mathrm{B}^{26}$. Interestingly, a similar pattern of SAC-independent mitotic arrest has also been described for polo-depleted S2 cells ${ }^{27}$. In cultured cells, human AurA phosphorylates the activation loop of Plk1, triggering entry into mitosis $^{28}$. In addition, as the CNS of polo mutants also displays $\mathrm{NB}$ amplification and tumour formation ${ }^{5}$, it is tempting to speculate that an AurA/Polo kinase cascade could control not only the timely entry into mitosis but also other mitotic events, including cyclin B degradation. APC/C subunits have many sites for in vivo phosphorylation by various kinases, including Plk1 and Aurora $\mathrm{A}^{29,30}$. A complex network of phosphorylation/ dephosphorylation events is probably required to promote the efficient degradation of APC/C substrates at the onset of anaphase, but Aurora A is undoubtedly a key player in this process.

In flies and human cell lines, Mad2 depletion accelerates the passage through mitosis ${ }^{14,31}$. Consistent with a role for Aurora A in the regulation of cyclin $B$ degradation, it has been suggested that the overproduction of Aurora A in one human cancer cell line overrides the SAC, leading to premature mitotic exit ${ }^{23}$. However, we found that NBs overexpressing Aurora A kinase had normal mitotic timing. Thus, by contrast to findings for human cancer lines, our results favour a model in which a pool of Aurora A functions normally downstream from or in parallel to the SAC, promoting the correctly timed and efficient degradation of cyclin $B$ in fly neural stem cells.

This study reveals, for the first time, that compromised Aurora A activity can prevent chromosome segregation defects and subsequent massive aneuploidy in a SAC-independent manner. This effect seems to be specific to aurA mutations, as sas-4 mutant NBs (which have similar spindle assembly and orientation defects), display severe impairment following SAC inactivation. For brain homeostasis, defective Aurora A activity therefore has disastrous consequences. First, it leads to the generation of many NB-like cells, due to a loss of cell polarity and to incorrect spindle orientation, leading to tumour formation. Second, the prolonged SAC-independent delay in mitosis enables aurA cells to avoid the potentially deleterious chromosome segregation defects observed for other mitotic mutants in the absence of the SAC.

Aurora A kinase is a target of several anticancer compounds currently undergoing preclinical trials. However, not all types of cancer seem to respond to Aurora A inhibitors ${ }^{32,33}$. We describe here an unexpected regulatory mechanism according to which Aurora A inhibition in fly neural stem cells leads to a delay in the progression of mitosis that is sufficiently long to allow the chromosomes to segregate correctly. It will therefore be of great interest to determine whether this mechanism is conserved in humans and whether it affects the relevance of Aurora A-based anticancer treatments in some types of tumours.

\footnotetext{
Methods

Live microscopy and immunofluorescence analysis. Third-instar larval brains were dissected in Schneider media and NBs were processed for live imaging or immunofluorescence analyses, as previously described ${ }^{12}$. Cyclin B-GFP degradation kinetics were analysed in individual NBs, with a published method for human cultured cells and fly $\mathrm{NBs}^{14,34}$. Images were acquired with a spinning-disk system mounted on an inverted microscope (Elipse Ti; Nikon) equipped with a $\times 601.4 \mathrm{NA}$ (numerical aperture) objective at $25^{\circ} \mathrm{C}$. Z series were acquired every $30 \mathrm{~s}$, with a sCMOS ORCA Flash 4.0 (Hamamatsu) controlled with MetaMorph
} acquisition software version X. Images were processed for quantification with
ImageJ software and are presented as maximum-intensity projections. GFP fluorescence was quantified for the whole cell. The signal was adjusted for background and for bleaching relative to the signal of a neighbouring non-mitotic cell (for which the signal was assumed to be constant). In the graphs, signal levels for the whole cell are displayed as normalized signals relative to the maximal intensity measured for the cell.

The brain squash analysis protocol for counting mitotic cells has been described elsewhere $\mathrm{e}^{35}$. Briefly, brains were dissected in PBS and incubated for $8 \mathrm{~min}$ in a $0.5 \%$ sodium citrate solution on a glass slide. The brains were then incubated for $30 \mathrm{~s}$ in $45 \%$ acetic acid and 2 min in $60 \%$ acetic acid, squashed with a clean, silicon-coated coverslip and frozen in liquid nitrogen. Once the coverslip had been removed, the preparations were briefly washed in PBS and mounted in Prolongold $+1 \mu \mathrm{g} \mathrm{ml}^{-1}$ 4,6-diamidino-2-phenylindole (Life Technologies). They were visualized with a Leica DMRXA2 microscope (objective $\times 63$, NA 1.32) and images were acquired with a CooSnap ES camera and Metamorph software (Roper Scientific). The images were processed with ImageJ. The mitotic index was calculated by determining the number of mitotic figures per optic field, every four fields. At least three brains were examined for quantification.

Antibodies and western blotting. The monoclonal YL1/2 rat anti-detyrosinated tubulin antibody (1:200) and the mouse monoclonal and rabbit polyclonal anti-phosphorylated histone H3 (Ser10) antibodies (1:500) were obtained from Millipore. The mouse monoclonal anti-cyclin A, anti-prospero and anti-cyclin B antibodies were purchased from the Developmental Studies Hybridoma Bank (1:10). The mouse monoclonal anti-FLAG $(1: 10,000)$ antibody was obtained from Stratagene. The rabbit anti-cyclin B antibody $(1: 10,000)$ has been described before $^{36}$ and was kindly provided by David Glover (University of Cambridge, UK) Polyclonal rabbit anti-actin (1:4,000) antibodies were obtained from Santa Cruz, and the rat anti-deadpan antibody $\left(1 \mu \mathrm{g} \mathrm{ml}^{-1}\right)$ was obtained from Abcam. The affinity-purified rabbit anti-Mad2 polyclonal antibody (1:500) was provided by David Sharp (Albert Einstein College of Medicine, New York). Goat secondary peroxidase-conjugated antibodies $(1: 5,000)$ were obtained from Jackson ImmunoResearch Laboratories and donkey Alexa Fluor-conjugated secondary antibodies $(1: 1,000)$ were obtained from Life Technologies. For western blotting, ECL reagent was purchased from Thermo Fisher Scientific. Some of the western blots were cropped and the original scans of the blots are provided in Supplementary Fig. 6.

Statistical analysis. Differences between data sets were assessed with a nonparametric test (Mann-Whitney-Wilcoxon), with values of $P<0.01$ considered significant.

Fly strains. Flies were maintained under standard conditions at $25^{\circ} \mathrm{C}$. Transgenic flies with the following genotypes were used for live imaging: H2A-RFP (Bloomington Stock Center), sas $-4^{s 2214}$ (ref. 4), cyclin B-GFP' ${ }^{21}$ mad2 ${ }^{P}$ (ref. 14), GFP-Mad2 (ref. 19) and H2A-GFP ${ }^{37}$ have been described elsewhere.

The aurA ${ }^{8839}$ allele (hereafter referred to as aurA) introduces a stop codon at position 377 in the open reading frame of the kinase ${ }^{7}$. The resulting mutant kinase has no detectable activity in vitro, like the K377R (dead) mutant $\left({ }^{7,38}\right)$. The aurA ${ }^{14641} /$ aurA $A^{17961}$ allelic combination has been described before and the corresponding fly stocks were provided by Chris Doe (University of Oregon) and Chen Yu Lee (University of Michigan) ${ }^{7}$. UAS-FLAG-AurA and UAS-FLAGAurA ${ }^{\mathrm{K} / \mathrm{R}}$ plasmids were constructed by the recombination of AurA and AurA $\mathrm{K}^{\mathrm{R}}$ donor vectors (described in ref. 38) in pPFW (Drosophila Genomic Resources Center) with Gateway technology (Life Technologies). Transgenic flies were obtained by $\mathrm{P}$ element-mediated transformation. The production of tagged proteins in the fly CNS was induced with the 69B-GAL4 strain ${ }^{12}$.

\section{References}

1. Homem, C. C. \& Knoblich, J. A. Drosophila neuroblasts: a model for stem cell biology. Development 139, 4297-4310 (2012).

2. Siller, K. H. \& Doe, C. Q. Spindle orientation during asymmetric cell division Nat. Cell. Biol. 11, 365-374 (2009).

3. Wodarz, A. \& Nathke, I. Cell polarity in development and cancer. Nat. Cell. Biol. 9, 1016-1024 (2007).

4. Basto, R. et al. Flies without centrioles. Cell 125, 1375-1386 (2006).

5. Castellanos, E., Dominguez, P. \& Gonzalez, C. Centrosome dysfunction in Drosophila neural stem cells causes tumors that are not due to genome instability. Curr. Biol. 18, 1209-1214 (2008).

6. Giet, R., Petretti, C. \& Prigent, C. Aurora kinases, aneuploidy and cancer, a coincidence or a real link? Trends Cell Biol. 15, 241-250 (2005).

7. Lee, C. Y. et al. Drosophila Aurora-A kinase inhibits neuroblast self-renewal by regulating aPKC/Numb cortical polarity and spindle orientation. Genes Dev. 20, 3464-3474 (2006).

8. Wang, H. et al. Aurora-A acts as a tumor suppressor and regulates self-renewal of Drosophila neuroblasts. Genes Dev. 20, 3453-3463 (2006). 
9. Wirtz-Peitz, F., Nishimura, T. \& Knoblich, J. A. Linking cell cycle to asymmetric division: Aurora-A phosphorylates the Par complex to regulate Numb localization. Cell 135, 161-173 (2008).

10. Giet, R. et al. Drosophila Aurora A kinase is required to localize D-TACC to centrosomes and to regulate astral microtubules. J. Cell Biol. 156, 437-451 (2002).

11. Glover, D. M., Leibowitz, M. H., McLean, D. A. \& Parry, H. Mutations in aurora prevent centrosome separation leading to the formation of monopolar spindles. Cell 81, 95-105 (1995).

12. Gallaud, E. et al. Ensconsin/Map7 promotes microtubule growth and centrosome separation in Drosophila neural stem cells. J. Cell Biol. 204, 1111-1121 (2014).

13. Janssen, A., Kops, G. J. \& Medema, R. H. Elevating the frequency of chromosome mis-segregation as a strategy to kill tumor cells. Proc. Natl Acad. Sci. USA 106, 19108-19113 (2009).

14. Buffin, E., Emre, D. \& Karess, R. E. Flies without a spindle checkpoint. Nat. Cell Biol. 9, 565-572 (2007).

15. Caussinus, E. \& Gonzalez, C. Induction of tumor growth by altered stem-cell asymmetric division in Drosophila melanogaster. Nat. Genet. 37, 1125-1129 (2005).

16. Rahmani, Z., Gagou, M. E., Lefebvre, C., Emre, D. \& Karess, R. E. Separating the spindle, checkpoint, and timer functions of BubR1. J. Cell Biol. 187, 597-605 (2009)

17. Johnston, C. A., Hirono, K., Prehoda, K. E. \& Doe, C. Q. Identification of an Aurora-A/PinsLINKER/Dlg spindle orientation pathway using induced cell polarity in S2 cells. Cell 138, 1150-1163 (2009).

18. Donaldson, M. M., Tavares, A. A., Ohkura, H., Deak, P. \& Glover, D. M. Metaphase arrest with centromere separation in polo mutants of Drosophila. J. Cell Biol. 153, 663-676 (2001).

19. Buffin, E., Lefebvre, C., Huang, J., Gagou, M. E. \& Karess, R. E. Recruitment of Mad2 to the kinetochore requires the Rod/Zw10 complex. Curr. Biol. 15, 856-861 (2005).

20. Katsani, K. R., Karess, R. E., Dostatni, N. \& Doye, V. In vivo dynamics of Drosophila nuclear envelope components. Mol. Biol. Cell 19, 3652-3666 (2008).

21. Huang, J. \& Raff, J. W. The disappearance of cyclin B at the end of mitosis is regulated spatially in Drosophila cells. EMBO J. 18, 2184-2195 (1999).

22. Lampson, M. A. \& Kapoor, T. M. The human mitotic checkpoint protein BubR1 regulates chromosome-spindle attachments. Nat. Cell Biol. 7, 93-98 (2005).

23. Anand, S., Penrhyn-Lowe, S. \& Venkitaraman, A. R. AURORA-A amplification overrides the mitotic spindle assembly checkpoint, inducing resistance to Taxol. Cancer Cell 3, 51-62 (2003).

24. Nagy, O. et al. lemmingA encodes the Apc11 subunit of the APC/C in Drosophila melanogaster that forms a ternary complex with the E2-C type ubiquitin conjugating enzyme, Vihar and Morula/Apc2. Cell Div. 7, 9 (2012).

25. Deak, P., Donaldson, M. \& Glover, D. M. Mutations in makos, a Drosophila gene encoding the Cdc27 subunit of the anaphase promoting complex, enhance centrosomal defects in polo and are suppressed by mutations in twins/ aar, which encodes a regulatory subunit of PP2A. J. Cell Sci. 116, 4147-4158 (2003).

26. Mathieu, J. et al. Aurora B and cyclin B have opposite effects on the timing of cytokinesis abscission in Drosophila germ cells and in vertebrate somatic cells. Dev. Cell 26, 250-265 (2013).

27. Conde, C. et al. Drosophila Polo regulates the spindle assembly checkpoint through Mps1-dependent BubR1 phosphorylation. EMBO J. 32, 1761-1777 (2013).

28. Macurek, L. et al. Polo-like kinase-1 is activated by aurora A to promote checkpoint recovery. Nature 455, 119-123 (2008).
29. Alexander, J. et al. Spatial exclusivity combined with positive and negative selection of phosphorylation motifs is the basis for context-dependent mitotic signaling. Sci. Signal. 4, ra42 (2011).

30. Kettenbach, A. N. et al. Quantitative phosphoproteomics identifies substrates and functional modules of Aurora and Polo-like kinase activities in mitotic cells. Sci. Signal. 4, rs5 (2011).

31. Meraldi, P., Draviam, V. M. \& Sorger, P. K. Timing and checkpoints in the regulation of mitotic progression. Dev. Cell 7, 45-60 (2004).

32. Malumbres, M. \& Perez de Castro, I. Aurora kinase A inhibitors: promising agents in antitumoral therapy. Expert Opin. Ther. Targets 18, 1377-1393 (2014)

33. Perez de Castro, I. et al. Requirements for Aurora-A in tissue regeneration and tumor development in adult mammals. Cancer Res. 73, 6804-6815 (2013).

34. Clute, P. \& Pines, J. Temporal and spatial control of cyclin B1 destruction in metaphase. Nat. Cell Biol. 1, 82-87 (1999).

35. Pimpinelli, S., Bonaccorsi, S., Fanti, L. \& Gatti, M. Preparation and orcein staining of mitotic chromosomes from Drosophila larval brain. Cold Spring Harb. Protoc. 2010, pdb prot5389 (2010).

36. Whitfield, W. G., Gonzalez, C., Maldonado-Codina, G. \& Glover, D. M. The A- and B-type cyclins of Drosophila are accumulated and destroyed in temporally distinct events that define separable phases of the G2-M transition. EMBO J. 9, 2563-2572 (1990).

37. Saint, R. \& Clarkson, M. Pictures in cell biology. A functional marker for Drosophila chromosomes in vivo. Trends Cell Biol. 10, 553 (2000).

38. Rome, P. et al. Aurora A contributes to p150(glued) phosphorylation and function during mitosis. J. Cell Biol. 189, 651-659 (2010).

\section{Acknowledgements}

This work was funded by the Ligue Nationale Contre le Cancer, l'Association de la Recherche contre le Cancer, Biosit and the University of Rennes 1. We thank D. Sharp, D. Buster, J. Raff, R. Basto, C. Doe, C.Y. Lee and D. Glover for fly stocks and antibodies. We thank the Microscopy Rennes Imaging Center and E. Gallaud for advices and help in designing experiments. R.C. is a doctoral fellow of the French Ministry of Research and the Association de la Recherche contre le Cancer.

\section{Author contributions}

R.C. and R.G. conceived the project and designed the experiments with the help of R.K.; R.C. performed the experiments with help from P.R., R.G., A.P. and L.R.-P.; L.R.-P., R.C., R.K. and R.G. interpreted the data; R.G. wrote the paper with the help of R.C., L.R.-P. and R.K

\section{Additional information}

Supplementary Information accompanies this paper at http://www.nature.com/ naturecommunications

Competing financial interests: The authors declare no competing financial interests

Reprints and permission information is available online at http://npg.nature.com/ reprintsandpermissions/

How to cite this article: Caous, R. et al. Spindle assembly checkpoint inactivation fails to suppress neuroblast tumour formation in aurA mutant Drosophila. Nat. Commun. 6:8879 doi: 10.1038/ncomms9879 (2015).

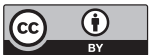

This work is licensed under a Creative Commons Attribution 4.0 International License. The images or other third party material in this article are included in the article's Creative Commons license, unless indicated otherwise in the credit line; if the material is not included under the Creative Commons license, users will need to obtain permission from the license holder to reproduce the material. To view a copy of this license, visit http://creativecommons.org/licenses/by/4.0/ 